\title{
Development of a Novel Antibacterial Hydrogel Scaffold Based on Guar Gum/poly (Methylvinylether-alt-Maleic Acid) Containing Cinnamaldehyde-Loaded Chitosan Nanoparticles
}

\author{
Leila Yavari Maroufi \\ Tabriz University of Medical Sciences \\ Marjan Ghorbani ( $\nabla$ ghorbani.marjan65@yahoo.com ) \\ Tabriz University of Medical Sciences
}

\section{Research Article}

Keywords: Composite hydrogels, Antibacterial activity, Guar gum, Poly(methylvinylether-alt-maleicacid), Cinnamaldehyde

Posted Date: March 31st, 2021

DOI: https://doi.org/10.21203/rs.3.rs-351185/v1

License: (c) (i) This work is licensed under a Creative Commons Attribution 4.0 International License. Read Full License 


\section{Abstract}

In this study, we produced a novel chemical cross-linked Guar gum/Poly(methylvinylether-alt-maleicacid) (GG/PMVE-MA) hydrogels with various blending weight ratio of GG, and PMVE-MAn (GG/P20, GG/P40, and GG/P70). These produced hydrogels were analyzed by fourier-transform infrared spectroscopy, scanning electron microscope, thermogravimetric analysis, swelling degree, and mechanical characteristics. The results demonstrated that with increasing PMVE-MAn content, thermal stability, swelling degree, and mechanical characteristics of hydrogels were improved. As a result, the GG/P70 hydrogel was selected as an optimal hydrogel. Moreover, MTT analyze indicated that these hydrogels were non-toxic and any reduction or stop of cells growth wasn't observed over time. Additionally, encapsulation of cinnamaldehyde (CA)-loaded chitosan nanoparticles CSNPs into optimal hydrogel formulation significantly $(\mathrm{P}<0.05)$ increased scavenging of DPPH radical about $60 \%$. In addition, the inhibition capability of GG/P70/CA-loaded CSNPs hydrogel against E. coli and S. areus, demonstrating that the hydrogel had high antibacterial and antioxidant activities. The general results showed that this composite hydrogel (GG/P70/CA-loaded CSNPs) could be useful for various applications such as drug delivery, tissue engineering and food industry.

\section{Introduction}

Newly, biopolymer-based hydrogels have been extensively identified as biomaterials due to their high biocompatibility, safety, bioactivity, and biodegradability [1-4]. Hydrogels are unique category of biomaterials with three-dimensional polymeric structures which can absorb high content of water or biological fluids, and display swelling behavior instead of dissolving in aqueous environment $[5,6]$. Hydrogels can be applied in many applications for example; drug delivery, tissue engineering, wound dressing and food packaging [7]. Guar gum is one of the suitable biopolymer for synthesis of hydrogels due to the low cost, hydrophilic nature and non-ionic polysaccharide which containing of galactose and mannose units [8-11]. However, the main limitation of GG for hydrogel fabrication is its high hydrophilic properties, and also reduction in viscosity $[12,13]$. These limitations of GG can be overcome by combining it with a synthetic/semi synthetic polymer, and chemical modification to produce an effective hydrogel [14]. Thereby, choosing the suitable synthetic polymer to overcome the limitations of GG is important.

Poly [(methyl vinyl ether)-alt-(maleic anhydride)] (PMVE-MAn) is a hydrophilic and biocompatible polymer which known as Gantrez ${ }^{\circledR A N}$. The hydrolyzed form of poly [methylvinylether-alt-maleic acid] (PMVE-MA) with the carboxylic acid functional groups can be used as support for drug delivery and cell growth. Hydrogels can be obtained by esterification grafting crosslinking reaction between maleic anhydride groups of PMVE-MAn and hydroxyl or amine groups of natural polymers [15]. Therefore, this synthetic polymer can act as a cross linker and create chemical cross-linking with GG.

$\mathrm{CA}$ is one of important constituent of cinnamon (Cinnamomum zeylanicum) which is existent in its essential oil. Because of high safety, and antibacterial effects against gram positive and gram negative 
bacteria, CA has many applications in several fields [16]. However, CA is unstable, volatile, and has a low water solubility which limits its application $[17,18]$. In order to overcome these limitations, CA can be encapsulated in natural safe nanoparticles [19]. Chitosan (CS) is widely applied as nontoxic material to produce nanoparticles as drug delivery system. CS is a natural, non-toxicity, biodegradable, biocompatible, and de-acetylated polysaccharide derived of chitin. Additionally, as a cationic polymer, it can be suitable for encapsulating active ingredient [20].

In this current study, the GG/PMVE-MA composite hydrogels were successfully produced. The various combination ratios of GG and PMVE-MAn were evaluated to assess their effects on the physicochemical properties of hydrogels. Chemical structure, morphology, thermal stability, mechanical properties, swelling ratio, and cell viability of hydrogel samples were determined. After that, CA was successfully encapsulated in the CS nanoparticles (CSNPS) and loaded in optimal GG/PMVE-MA composite hydrogels. Additionally, the particle size of CSNPs, antioxidant activity, antibacterial activity of optimal hydrogel containing CA-loaded CSNPs were assessed. This paper presents a scientific report of developed GG/PMVE-MA/CA-loaded CSNPs composite hydrogels for use in different industrial applications.

\section{Materials And Methods}

\subsection{Materials}

The GG, PMVE-MAn ( $\left.\mathrm{M}_{\mathrm{w}}=216 \mathrm{kDa}\right), \mathrm{CS}$ (from shrimp shells, > 75\% deacetylated), CA ( $\left.\geq 95 \%\right)$, Pentasodium tripolyphosphate (TPP), thiazolyl blue tetrazolium bromide (MTT), fetal bovine serum (FBS), trypsin-EDTA, RPMI 1640 medium, Dimethyl sulfoxide (DMSO), and Phosphate buffered saline (PBS) were purchased from Sigma-Aldrich (St. Lo., USA). DPPH (1,1-Diphenyl-2-picrylhydrazyl), 1\% glacial acetic acid, Ethanol 96\%, Tween 80\%, and Muller Hinton Agar were purchased from Merck Chemical Co. (Darmstadt, Germany). The mouse embryonic fibroblast cell line (NIH-3T3), and Stock cultures, Escherichia coli (PTCC-1270), Staphylococcus aureus (PTCC-1112) were purchased from Pasture Institute (Iran).

\subsection{Synthesis of CSNPs and CA-loaded CSNPS}

CA-loaded CSNPs were prepared using ionic gelation assay with minor change [21]. In briefly, CA (0.3 mL, $1 \mathrm{v} / \mathrm{v} \%)$ was added slowly into preheated CS solution $(0.4 \% \mathrm{w} / \mathrm{v})$ under magnetic stirring. After that, TPP solution $(0.3 \% \mathrm{w} / \mathrm{v})$ was added gradually into CA-CS solution under magnetic stirring (at $800 \mathrm{rpm})$ for 20 min at room temperature to obtain CA-loaded CSNPs. Tween $80 \%$ was used as a surfactant. The nanodispersion was then centrifuged at $10,000 \times \mathrm{g}$ for $1 \mathrm{~h}$ and washed by distilled water (4 times) to remove the unloaded CA.

\subsection{Particle size, zeta potential and encapsulation efficiency (EE) studies}


The particle size and zeta potential of CSNPs were calculated with a dynamic light scattering (DLS) (Zetasizer Nano-ZS90, Malvern Instruments Ltd., Worcestershire, UK) under vacuum condition. All evaluations were performed at room temperature. The EE \% of CA-loaded CSNPs was calculated by UVVis spectrophotometer (Spectrum SP-UV500DB) at $285 \mathrm{~nm}$.

$$
\mathrm{EE}(\%)=\frac{\text { Total } C A \text { loaded }- \text { Non encapsulated } C A}{\text { Total } C A \text { loaded }} \times 100
$$

\subsection{Preparation of composite hydrogels}

GG $(0.2 \% \mathrm{w} / \mathrm{v})$ /PMVE-MA ( $2 \% \mathrm{w} / \mathrm{v})$ hydrogel samples were prepared as follows; aqueous solutions comprising various weight ratios of GG, and PMVE-MAn were put in 24-well plates. Solutions were freezedried for $72 \mathrm{~h}$. The final hydrogel samples were taken from the 24-well plates. The freeze-dried hydrogel samples were put into an oven at $75^{\circ} \mathrm{C}$ for 3 days and $8 \mathrm{~h}$ for each day. The final obtained hydrogels were cited in a high content of water for $24 \mathrm{~h}$. Finally, these hydrogel samples were frozen in liquid nitrogen and after that freeze-dried for $48 \mathrm{~h}$.

Table 1. Preparation chart of GG/P (Guar Gum/PMVE-MA) hydrogels.

\begin{tabular}{|lll|}
\hline Hydrogel Sample Code & GG & PMVE-MAn (2\% w/v) \\
\hline GG/P20 & $(0.2 \% \mathrm{w} / \mathrm{v})$ & \\
\hline GG/P40 & 80 & 20 \\
\hline GG/P70 & 60 & 40 \\
\hline
\end{tabular}

\subsection{Characterization}

FTIR spectra were recorded for GG, PMVE-MA, and final hydrogels with Thermo Avatar 370 spectrometer (Tensor27, Bruker Co., Ettlingen, Germany) in transmittance mode amongst $400-4000 \mathrm{~cm}^{-1}$. The morphology of hydrogels was analyzed by a scanning electron microscopy (SEM, Hitachi High-Tech HITACHI, Tokyo, Japan). To investigate the thermal stability of produced hydrogels, thermogravimetric analysis (TGA) was performed with a LINSEIS SPA PT 1600 device (Germany) at a range of $20-600^{\circ} \mathrm{C}$, at a heating rate of $10^{\circ} \mathrm{C} / \mathrm{min}$ and under $\mathrm{N}_{2}$.

\subsection{Swelling degree}

To measure the swelling degree of hydrogel samples, gravimetric analysis was performed [22]. In short, at first the dry weight of the hydrogels was measured $\left(W_{d}\right)$, after that the hydrogels were immersed in $30 \mathrm{ml}$ of PBS solution at room temperature. After that, the swollen hydrogels were weighed after 2, 4, 7, 10, 12, $24,36,72$, and $96 \mathrm{~h}\left(\mathrm{~W}_{\mathrm{s}}\right)$. The swelling degree $(\mathrm{SD})$ was measured according with the Eq: 
$\mathrm{SD}(\%)=\frac{\mathrm{W}_{s}-\mathrm{W}_{d}}{\mathrm{~W}_{d}} \times 100$

\subsection{Mechanical Characteristics}

Using a universal testing system (INSTRON series 3366), the mechanical properties of the samples were investigated. The initial elastic modulus was calculated from the slope of the initial linear segment of stresss-train curves, Tensile strength was taken as the maximum force divided by the minimum crosssectional area of the specimen to find a precise tensile strength. The strain value corresponding to the maximum stress was calculated as a strain at break.

\subsection{Cell viability study}

The MTT technique was accomplished to explore the cell viability of hydrogel samples [23-25]. In summary, the hydrogel samples were cut into $12 \mathrm{~mm}^{2}$ and immersed in $75 \%$ ethanol for $60 \mathrm{~min}$ to be sterilized. After that, $1 \mathrm{~mL}$ of cell suspension including $5 \times 10^{4}$ cells was seeded onto sterilized hydrogel samples and incubated in RPMI- 1640 including $10 \% \mathrm{FBS}$ at $37^{\circ} \mathrm{C}$ with $5 \% \mathrm{CO}_{2}$. After $1,3,5$ and 7 days, $200 \mu \mathrm{L}$ DMSO was added to dissolve formazan crystals formed inside cells. Then, absorbance was determined using spectrophotometer (UV-2550, Shimadzu, Japan) at $570 \mathrm{~nm}$.

\subsection{Antioxidant capacity}

The antioxidant capacity of the optimal hydrogel and optimal hydrogel containing CA-loaded CSNPs was calculated with the DPPH radical scavenging assay during $9 \mathrm{~h}$ of incubation [26]. In summary, $0.1 \mathrm{mM}$ of DPPH solution in ethanol was prepared. Next, $1 \mathrm{~mL}$ of DPPH solution was added to $3 \mathrm{~mL}$ of solution containing hydrogel and hydrogel/CA-loaded CSNPs. Finally, after $30 \mathrm{~min}$, the absorbance was measured at $517 \mathrm{~nm}$ assessed with a UV-Vis spectrophotometer (Spectrum SP-UV500DB) after 3, 6, and 9h. The DPPH radical scavenging was calculated with the Eq:

$$
\text { DPPH radical scavenging }(\%)=\frac{A b s_{D P P H}-A b s_{\text {extract }}}{A b s_{D P P H}} \times 100
$$

Where Abs $s_{\text {DPPH }}$ was the absorbance of DPPH ethanolic solution and Abs $_{\text {extract }}$ was the absorbance of the hydrogels.

\subsection{Antibacterial activity}

Antibacterial activities of the optimal hydrogel and optimal hydrogel containing CA-loaded CSNPS against $S$. areus and $E$. coli bacteria were measured using disk diffusion methods [8]. In short, $3-5 \mathrm{~mm}$ disk of hydrogel sample was exposed on Mueller-Hinton agar plate that cultured by $1.5 \times 10^{8} \mathrm{CFU} / \mathrm{mL}$ concentration of each bacterial suspension. After $24 \mathrm{~h}$, inhibition of bacterial growth area about the hydrogel sample disk was determined and expressed. 


\subsection{Statistical analysis}

Statistical differences $(P<0.05)$ were analyzed using one-way ANOVA followed by Tukey's test for multiple comparisons by GraphPad Prism 5. Obtained results were expressed as mean \pm standard deviation (SD). The level of significance was considered $\mathrm{P}<0.05$.

\section{Results And Discussion}

\subsection{Synthesis of cross-linked GG/ PMVE-MA hydrogels}

To synthesize the cross-linked hydrogels, the aqueous solutions comprising various concentrations and weight ratios of GG and PMVE-MAn were mixed. The mixed solutions were freeze-dried for $72 \mathrm{~h}$. After that, resulting hydrogel samples were transferred into an oven at $75^{\circ} \mathrm{C}$ for 3 days, and $8 \mathrm{~h}$ at each day. The final obtained hydrogels were cited for $24 \mathrm{~h}$ in a high content of water. Finally, these hydrogel samples were frozen in liquid nitrogen and after that freeze-dried. During this process, an esterification reaction occurs between the hydroxyl groups of GG and maleic anhydride groups of PMVE-MAn [27]. Thereby, the cross-linked GG/PMVE-MA (GG/P) hydrogels were produced.

\subsection{Particle size and zeta potential studies}

The formation and size distribution of CA-loaded CSNPs was investigated using DLS analysis. The size of CA-loaded CSNPs ranged from 100 to $338.21 \mathrm{~nm}$ with a mean particle size of $218 \mathrm{~nm}$ was shown in the Fig. 1. The CA-loaded CSNPs nanodispersion revealed a poly dispersity index (PDI) of 0.277 and zeta potential of $36 \mathrm{mV}$. The EE\% was calculated using a standard curve of CA. The EE (\%) of CA-loaded CSNPs was observed to be $69.25 \pm 1.2 \%$.

\subsection{FTIR characterization}

The FTIR spectra of GG, PMVE-MAn, and GG/PMVE-MA hydrogels were demonstrated in Fig. 2. The FTIR spectrum of GG showed an extensive peak at $3539 \mathrm{~cm}^{-1}$ attributed to the $\mathrm{OH}$ stretching and the peak at $2920 \mathrm{~cm}^{-1}$ related to the $\mathrm{CH}$ stretching. Also, the peak at $1641 \mathrm{~cm}^{-1}$ attributed to $\mathrm{C}-\mathrm{OH}$ vibrations [8]. The FTIR spectrum of PMVE-MAn indicates the attendance of a peak at $1107 \mathrm{~cm}^{-1}$ owing to $\mathrm{C}-\mathrm{O}-\mathrm{C}$ group. The peak at $1701 \mathrm{~cm}^{-1}$ attributed to the carbonyl groups of acidic groups of PMVE-MA [28, 29]. In the FTIR spectrum of GG/PMVE-MA hydrogels, the peak at $1701 \mathrm{~cm}^{-1}$ which related to the carbonyl groups of PMVE-MAn was shifted to $1732 \mathrm{~cm}^{-1}$, which indicates the occurrence of a reaction between PMVEMAn and GG. Additionally, the peak at $1641 \mathrm{~cm}^{-1}$ which related to C-OH vibrations of GG, was shifted to $1648 \mathrm{~cm}^{-1}$, that approved the occurrence of ester and anhydride bonds in the GG/PMVE-MA hydrogels. Moreover, after the addition of CNPs to the hydrogel network, a little shifting of position in the characteristic bands of $\mathrm{C}-\mathrm{OH}$ and carbonyl groups (at $1648 \mathrm{~cm}^{-1}$ to $1638 \mathrm{~cm}^{-1}$ and $1732 \mathrm{~cm}^{-1}$ to $1730 \mathrm{~cm}$ ${ }^{1}$ ) for $\mathrm{CNPs} /$ hydrogel due to the hydrogen bond formation between the polymeric network of hydrogel and the $-\mathrm{OH}$ and $-\mathrm{NH} 2$ groups of CNPs. 


\subsection{Morphological studies}

The morphology of GG/PMVE-MA hydrogel samples was investigated with SEM analysis. The SEM results of freeze-dried hydrogels with various combination ratios of GG, and PMVE-MAn was demonstrated in Fig. 3. As is clear in Fig. 3, the structure of the all hydrogel samples was porous. However, the changes of the PMVE-MAn content seem to affect the morphology of the hydrogels. The hydrogels containing a large content of PMVE-MAn (GG/P70 sample) demonstrated a spongy, and dense structure with smaller pores. This result can be related to the increasing of cross-linking density resulted from the occurrence of esterification and anhydrization reaction in the GG/PMVE-MA hydrogels. The similar results can be seen in previous studies $[27,30,31]$.

\subsection{TGA analysis}

Fig. 4, displayed the TGA thermograms of GG/PMVE-MA hydrogels with various blending weight ratio of GG, and PMVE-MAn. As can realize from this Fig, the first stage weight loss of hydrogels is between 50 and $150^{\circ} \mathrm{C}$ which is related to the loss of free and bound water adsorbed in the structure of hydrogels. The second stage of weight loss occurred at the range of $150-380^{\circ} \mathrm{C}$ which is associated to the chemical decomposition of the GG [32]. Weight loss in the second stage for hydrogel sample with high content of GG (GG/P20) occured with more rate. By increasing the content of PMVE-MAn in the structure of GG/PMVE-MA hydrogels the thermal stability was improved owing to the chemical crosslinking reaction between the -OH groups of the GG and the carbonyl groups of the PMVE-MAn $[27,28]$.

\subsection{Swelling behavior}

The swelling degree of the hydrogel samples with various blending weight ratios of GG, and PMVE-MAn was determined in PBS (pH 7.4) solution at $37^{\circ} \mathrm{C}$ after 2, 4, 7, 10, 12, 24, 36, 72, and 96 h. Fig. 5 presented the swelling profile of hydrogel samples. As can be seen from this Fig, the swelling ratio of hydrogel samples increased over time with increasing the PMVE-MAn content (about 400\%). This result could be related to the increased chemical crosslinking reaction between the $-\mathrm{OH}$ groups of the $\mathrm{GG}$ and the $-\mathrm{C}-\mathrm{O}$ C- groups of the PMVE-MAn and number of pores, that previous studies were confirmed the importance of increasing the chemical crosslinking between polymers in relation of increasing the swelling ratio $[9,27$, $33,34]$.

\subsection{Mechanical characterization}

The mechanical characteristics of hydrogels are one of the important parameters for evaluating their durability and resistance to external forces [35]. The mechanical characteristics of the hydrogels were investigated by tensile strength (TS), elongation at break (EAB), and elastic modulus [36]. Since pure GGbased hydrogels have weak and undesirable mechanical properties, improving, and reinforcing these properties by adding synthetic polymer and or cross-linking can be very effective [37]. In this paper, the mechanical characteristics of hydrogels were slightly improved by increasing the content of PMVE-MAn. As shown in Fig. 6, the order of the mechanical characteristics of GG/PMVE-MA with different blending 
weight ratio of GG, and PMVE-MAn was found as: GG/P70 > GG/P40> GG/P20. The obtained results showed that with more PMVE-MAn content, the mechanical characteristics of the GG/PMVE-MA hydrogels were improved. So that, the TS, EAB, and elastic modulus of GG/PMVE-MA hydrogel with ratio of GG/P70 as the optimal hydrogel reached at $\sim 90 \pm 4.2 \mathrm{MPa}, 83 \pm 1.75 \%$, and $90.2 \pm 1.4 \mathrm{KPa}$, respectively. These results were related to the degree of chemical crosslinking between the $-\mathrm{OH}$ groups of GG and carbonyl groups of PMVE-MAn which had hardened the hydrogel structure. This result is consistent with the results of other research in this area [38-41].

\subsection{Cell viability}

The biocompatibility of the hydrogel samples for cell growth was investigated using MTT assay. Fig. 7, illustrated the cell viability of NIH3T3 fibroblast cells in the attendance of the GG/PMVE-MA hydrogels with different blending weight ratios of GG, and PMVE-MAn (GG/P20, GG/P40, and GG/P70). As realized from this figure, all hydrogel samples were appropriate for cell growth and survival and the cell growth wasn't stop or even decrease. This obtained result could be related to the safety of GG, and PMVE-MAn which have the capability to interactions between growth factors and other Extra Cellular Matrix (ECM) proteins which can enhanced cellular activities $[42,43]$.

\subsection{Antioxidant capacity}

To induce antioxidant and antimicrobial capability for the produced GG/PMVE-MA hydrogels, CA-loaded CSNPs was encapsulated in the optimal hydrogel formulation (GG/P70). In this regard, the hydrogel extract exposed to DPPH radicals and the resulting adsorption was determined at $517 \mathrm{~nm}$ after different reaction times $(3,6$, and $9 \mathrm{~h})$ to assess the antioxidant capacity. The results of DPPH radical scavenging activity assay were displayed in the Fig. 8. DPPH method indicated that the DPPH radical scavenging rate of blank hydrogel was only $6.21 \pm 1.23 \%$, and also wasn't observed significant changes in the DPPH radical scavenging rate with increasing reaction time, indicating that its antioxidant capacity was low. Whereas, the encapsulation of CA-loaded CSNPs into the optimal hydrogel formulations (GG/P70) induced the antioxidant activity and increased the DPPH radical scavenging rate. This result is consistent with the results of other research in this area [44-49]. Also, the DPPH radical scavenging rate of hydrogel containing CA-loaded CSNPs increased with increasing reaction time that can be associated to the increased CA release, resulting in increased reaction rate and DPPH radical scavenging.

\subsection{Antibacterial activity}

To evaluate the antibacterial effect of optimal hydrogel (GG/P70) containing CA-loaded CSNPs, the agar disc diffusion assay was used to found the amount of clear zone obtained from a circular hydrogel sample disk. The obtained results indicated that the control sample (GG/P70) did not inhibit the growth of the pathogenic bacteria. As shown in Fig. 9, E. coli (gram negative bacteria), and S. aureus (gram positive bacteria) were sensitive to CA essential oil. In this content, the diameter of the clear zone is 14.3 $\pm 1.12 \mathrm{~mm}$ for $S$. aureus. There are similar results in many previous studies confirmed our results $[18,44$, 
$46,50,51]$. Since, the gram-negative bacteria have a more complex wall structure, so, the gram-negative bacteria outer mats prevents the penetration of CS-loaded CSNPs into the bacterial cell.

\section{Conclusion}

The chemical cross-linked GG/PMVE-MA hydrogels with various combination weight ratios (GG/P20, GG/P40, and GG/P70) were successfully produced. The biological and physicochemical properties of produced hydrogels were investigated using different analysis. The hydrogel with more content of PMVEMAn (GG/P70) demonstrated the high cross-linking density and spongy structure with smaller and more pores. Also, by enhancing the content of PMVE-MAn, mechanical properties and swelling ratio significantly $(P<0.05)$ were improved. Additionally, the particle size of CA-loaded CSNPs displayed $218 \mathrm{~nm}$ with poly dispersity index of 0.277 and zeta potential of $36 \mathrm{mV}$. Subsequently, by encapsulation of CAloaded CSNPs into the optimal hydrogel formulation (GG/P70), antioxidant and antimicrobial activity were induced in the developed hydrogel. The obtained results of antioxidant and antimicrobial studies demonstrated that the hydrogel (GG/P70/CA-loaded CSNPs) had high antibacterial and antioxidant activities. Overall results showed that this composite hydrogel (GG/P70/CA-loaded CSNPs) can be useful for various applications such as drug delivery, tissue engineering and food industry.

\section{Declarations}

\section{Acknowledgment}

This research was supported with the Nutrition Research Center; Tabriz University of Medical Sciences.

\section{References}

1. Nezhad-Mokhtari P, Ghorbani M, Roshangar L, Soleimani Rad J (2019) A review on the construction of hydrogel scaffolds by various chemically techniques for tissue engineering. Eur Polym J 117:6476 . doi: 10.1016/j.eurpolymj.2019.05.004

2. Huang B, Liu M, Zhou C (2017) Chitosan composite hydrogels reinforced with natural clay nanotubes agents. Carbohydr Polym 175:689-698 . doi: 10.1016/j.carbpol.2017.08.039

3. Ghorbani M, Nezhad-Mokhtari P, Ramazani S (2020) Aloe vera-loaded nanofibrous scaffold based on Zein/Polycaprolactone/Collagen for wound healing. Int J Biol Macromol

4. Nezhad-Mokhtari P, Ghorbani M, Roshangar L, Soleimani Rad J (2019) Chemical gelling of hydrogelsbased biological macromolecules for tissue engineering: Photo- and enzymatic-crosslinking methods. Int J Biol Macromol 139:760-772 . doi: 10.1016/j.ijbiomac.2019.08.047

5. Wahid F, Wang H, Lu Y, et al (2017) Preparation , characterization and antibacterial applications of carboxymethyl. Int J Biol Macromol. doi: 10.1016/j.ijbiomac.2017.03.132 
6. Kalia S, Choudhury AR (2019) International Journal of Biological Macromolecules Synthesis and rheological studies of a novel composite hydrogel of xanthan, gellan and pullulan. Int J Biol Macromol 137:475-482 . doi: 10.1016/j.ijbiomac.2019.06.212

7. Wahid F, Hu X, Chu L, et al (2018) PT. Int J Biol Macromol \#pagerange\# . doi: 10.1016/j.ijbiomac.2018.10.105

8. Yavari Maroufi L, Ghorbani M, Tabibiazar M (2020) A Gelatin-Based Film Reinforced by Covalent Interaction with Oxidized Guar Gum Containing Green Tea Extract as an Active Food Packaging System. Food Bioprocess Technol 13:1633-1644 . doi: 10.1007/s11947-020-02509-7

9. Nezhad-mokhtari P, Ghorbani M, Yazdani N (2020) Jo ur na of. Int J Biol Macromol. doi: 10.1016/j.ijbiomac.2020.11.103

10. Sharma R, Kaith BS, Kalia S, et al (2015) Biodegradable and conducting hydrogels based on Guar gum polysaccharide for antibacterial and dye removal applications. 162:

11. Thombare N, Mishra S, Siddiqui MZ, et al (2018) PT Effect on water retention capacity of soil SC. Carbohydr Polym. doi: 10.1016/j.carbpol.2018.01.018

12. Li X (2008) Synthesis and properties of thermo-responsive guar gum / poly ( $\mathrm{N}$-isopropylacrylamide ) interpenetrating polymer network hydrogels. 71:394-402 . doi: 10.1016/j.carbpol.2007.06.005

13. Dai L, Zhang L, Wang B, et al (2017) Multifunctional self-assembling hydrogel from guar gum. Chem Eng J. doi: 10.1016/j.cej.2017.08.041

14. Maity J, Ray SK (2016) Enhanced adsorption of $\mathrm{Cr}(\mathrm{VI})$ from water by guar gum based composite hydrogels. Int J Biol Macromol. doi: 10.1016/j.jibiomac.2016.04.036

15. Ma X, Zhou N, Zhang T, et al (2017) Self-healing pH-sensitive poly [( methyl vinyl ether ) - alt - (maleic acid )] -based supramolecular hydrogels formed by inclusion complexation between cyclodextrin and adamantane. Mater Sci Eng C 73:357-365 . doi: 10.1016/j.msec.2016.12.039

16. Naveena BM, Muthukumar M, Sen AR, et al (2014) USE OF CINNAMALDEHYDE AS A POTENTIAL ANTIOXIDANT IN GROUND SPENT HEN MEAT. 38:1911-1917 . doi: 10.1111/jfpp.12163

17. Zhang L, Huang C, Xu Y, et al (2020) International Journal of Biological Macromolecules Synthesis and characterization of antibacterial polylactic acid fi Im incorporated with cinnamaldehyde inclusions for fruit packaging. Int J Biol Macromol 164:4547-4555 . doi: 10.1016/j.ijbiomac.2020.09.065

18. Gadkari RR, Suwalka S, Yogi MR, et al (2019) ur na I P re of. Carbohydr Polym 115298 . doi: 10.1016/j.carbpol.2019.115298 
19. Shao P, Liu Y, Ritzoulis C, Niu B (2019) Preparation of zein nano fi bers with cinnamaldehyde encapsulated in surfactants at critical micelle concentration for active food packaging. Food Packag Shelf Life 22:100385 . doi: 10.1016/j.fpsl.2019.100385

20. Ji M, Sun X, Guo X, et al (2018) Green synthesis, characterization and in vitro release of cinnamaldehyde/sodium alginate/chitosan nanoparticles. Food Hydrocoll. doi: 10.1016/j.foodhyd.2018.12.027

21. Rejinold NS, Muthunarayanan M, Muthuchelian K, et al (2011) Saponin-loaded chitosan nanoparticles and their cytotoxicity to cancer cell lines in vitro. 84:407-416 . doi: 10.1016/j.carbpol.2010.11.056

22. Zhang L, Liu J, Zheng X, et al (2019) SC. Carbohydr Polym. doi: 10.1016/j.carbpol.2019.04.004

23. Akrami-Hasan-Kohal M, Ghorbani M, Mahmoodzadeh F, Nikzad B (2020) Development of reinforced aldehyde-modified kappa-carrageenan/gelatin film by incorporation of halloysite nanotubes for biomedical applications. Int J Biol Macromol

24. Ghorbani M, Roshangar L, Soleimani J (2020) Development of reinforced chitosan / pectin sca ff old by using the cellulose nanocrystals as nano fi llers: An injectable hydrogel for tissue engineering. Eur Polym J 130:109697 . doi: 10.1016/j.eurpolymj.2020.109697

25. Ghorbani M, Hamishehkar $\mathrm{H}$, Hajipour $\mathrm{H}$, et al (2016) Ternary-responsive magnetic nanocarriers for targeted delivery of thiol-containing anticancer drugs. New J Chem 40:3561-3570 . doi: $10.1039 /$ C5NJ03602J

26. Zhang S, Hou J, Yuan Q, et al (2019) Arginine derivatives assist dopamine-hyaluronic acid hybrid hydrogels to have enhanced antioxidant activity for wound healing Arginine derivatives assist dopamine-hyaluronic acid hybrid hydrogels to have enhanced antioxidant activity for wound healing. Chem Eng J 123775 . doi: 10.1016/j.cej.2019.123775

27. Bucatariu S, Constantin M, Rusu D, et al (2020) Jo ur I P. Int J Biol Macromol. doi: 10.1016/j.ijbiomac.2020.10.095

28. Rohatgi CV, Dutta NK, Choudhury NR (2015) Separator Membrane from Crosslinked Poly(Vinyl Alcohol) and Poly(Methyl Vinyl Ether-alt-Maleic Anhydride). 398-414 . doi: 10.3390/nano5020398

29. Larrañeta E, Barturen L, Ervine M, Donnelly RF (2018) Hydrogels based on poly ( methyl vinyl etherco-maleic acid ) and Tween 85 for sustained delivery of hydrophobic drugs. Int J Pharm 538:147158 . doi: 10.1016/j.jpharm.2018.01.025

30. Zhang L, Liu J, Zheng X, et al (2019) Pullulan dialdehyde crosslinked gelatin hydrogels with high strength for biomedical applications. Carbohydr Polym 216:45-53 . doi:

10.1016/j.carbpol.2019.04.004

Page $11 / 21$ 
31. Zhang Q, Hu XM, Wu MY, et al (2019) Synthesis and performance characterization of poly ( vinyl alcohol ) -xanthan gum composite hydrogel. 136:34-43 . doi:

10.1016/j.reactfunctpolym.2019.01.002

32. Ahammed S, Liu F, Khin MNOE, et al (2020) Improvement of the water resistance and ductility of gelatin film by zein. Food Hydrocoll 105804

33. Poorna KS V, Singh A, Rathore A, Kumar A (2016) Novel cross linked guar gum -g- poly ( acrylate ) porous superabsorbent hydrogels: Characterization and swelling behaviour in different environments. Carbohydr Polym 149:175-185 . doi: 10.1016/j.carbpol.2016.04.077

34. de Souza AG, Cesco CT, de Lima GF, et al (2019) Arabic gum-based composite hydrogels reinforced with eucalyptus and pinus residues for controlled phosphorus release. Int J Biol Macromol 140:3342 . doi: 10.1016/j.ijbiomac.2019.08.106

35. Hassan A, Bilal M, Niazi K, et al (2018) Development of Anti-bacterial PVA / Starch Based Hydrogel Membrane for Wound Dressing. J Polym Environ 26:235-243 . doi: 10.1007/s10924-017-0944-2

36. Goodarzi H, Jadidi K, Pourmotabed S, et al (2019) International Journal of Biological

Macromolecules Preparation and in vitro characterization of cross-linked collagen - gelatin hydrogel using EDC / NHS for corneal tissue engineering applications. 126:620-632 . doi: 10.1016/j.ijbiomac.2018.12.125

37. Maity J, Ray SK (2016) International Journal of Biological Macromolecules Enhanced adsorption of $\mathrm{Cr}(\mathrm{VI})$ from water by guar gum based composite hydrogels. Int J Biol Macromol 89:246-255 . doi: 10.1016/j.ijbiomac.2016.04.036

38. Wang L, Lin L, Guo Y, et al (2020) Enhanced functional properties of nanocomposite film incorporated with EGCG-loaded dialdehyde glucomannan/gelatin matrix for food packaging. Food Hydrocoll 108:105863 . doi: 10.1016/j.foodhyd.2020.105863

39. Dash R, Foston M, Ragauskas AJ (2013) Improving the mechanical and thermal properties of gelatin hydrogels cross-linked by cellulose nanowhiskers. Carbohydr Polym 91:638-645

40. Zerbinati N, Esposito C, Cipolla G, et al Thuong 10 , Fabiola Sangalli², Raffaele Rauso 11 , Torello Lotti 12. doi: $10.1111 /$ dth.13747

41. Xie Y, Liao X, Zhang J, et al (2018) International Journal of Biological Macromolecules Novel chitosan hydrogels reinforced by silver nanoparticles with ultrahigh mechanical and high antibacterial properties for accelerating wound healing. Int J Biol Macromol 119:402-412 . doi: 10.1016/j.ijbiomac.2018.07.060 
42. Salbach J, Rachner TD, Rauner M, et al (2012) Regenerative potential of glycosaminoglycans for skin and bone. J Mol Med 90:625-635

43. Science B (2014) Mineralized collagen scaffolds induce hMSC osteogenesis and matrix remodeling. doi: $10.1039 / \times 0 x x 00000 x$

44. Manukumar HM, Umesha S (2017) PT US CR. Food Res Int. doi: 10.1016/j.foodres.2017.09.095

45. Mata MAL, Ruiz S, José C, et al (2017) Mechanical , Barrier and Antioxidant Properties of Chitosan Films Incorporating Cinnamaldehyde. J Polym Environ 0:0 . doi: 10.1007/s10924-017-0961-1

46. Hosseini SF, Ghaderi J (2021) Food Hydrocolloids trans- Cinnamaldehyde-doped quadripartite biopolymeric films: Rheological behavior of film-forming solutions and biofunctional performance of films. 112: . doi: 10.1016/j.foodhyd.2020.106339

47. Lopes R, Freitas M, Andrade T, et al (2016) Chitosan coating with trans-cinnamaldehyde improves structural integrity and antioxidant metabolism of fresh-cut melon Postharvest Biology and Technology Chitosan coating with trans -cinnamaldehyde improves structural integrity and antioxidant metabolism . Postharvest Biol Technol 113:29-39 . doi:

10.1016/j.postharvbio.2015.11.004

48. Villegas C, Torres A, Rios M, et al (2017) Supercritical impregnation of cinnamaldehyde into polylactic acid as a route to develop antibacterial food packaging materials. Food Res Int. doi: 10.1016/j.foodres.2017.06.031

49. Norton IT, Batchelor HK, Luis JP, et al (2017) Food Hydrocolloids Physico-chemical , antimicrobial and antioxidant properties of gelatin- chitosan based $\mathrm{fi} I \mathrm{Ims}$ loaded with nanoemulsions encapsulating active compounds rez-C o. doi: 10.1016/j.foodhyd.2017.12.012

50. Chen W, Cheng F, Swing CJ, et al (2019) Modulation e ff ect of core-wall ratio on the stability and antibacterial activity of cinnamaldehyde liposomes. Chem Phys Lipids 223:104790 . doi: 10.1016/j.chemphyslip.2019.104790

51. Ji M, Wu J, Sun X, et al (2021) Physical properties and bioactivities of fish gelatin films incorporated with cinnamaldehyde-loaded nanoemulsions and vitamin C. LWT 135:110103 . doi: 10.1016/j.Iwt.2020.110103

\section{Figures}



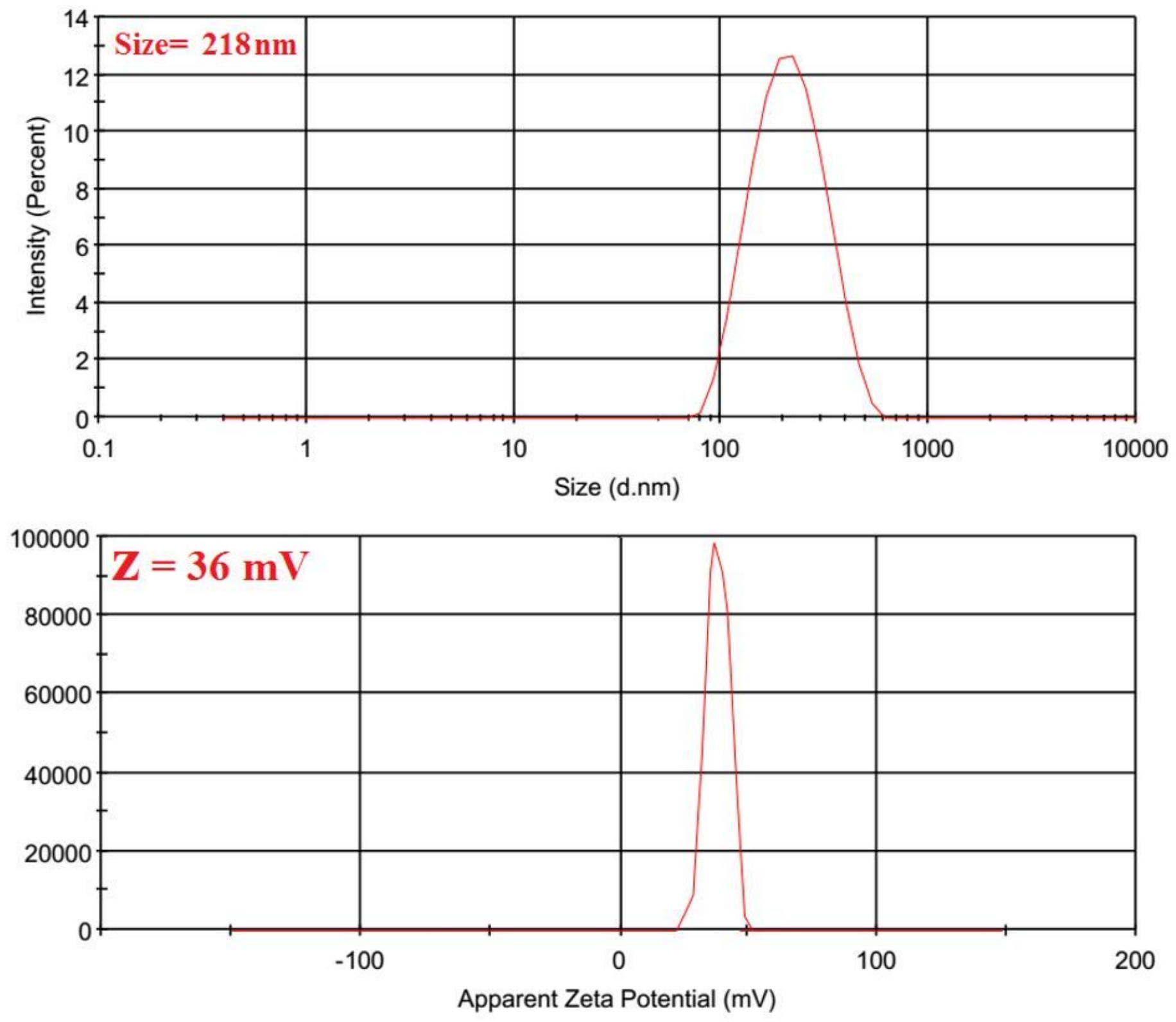

Figure 1

Particle size and zeta potential of cinnamaldehyde-loaded chitosan nanoparticles (CA-loaded CSNPs). 


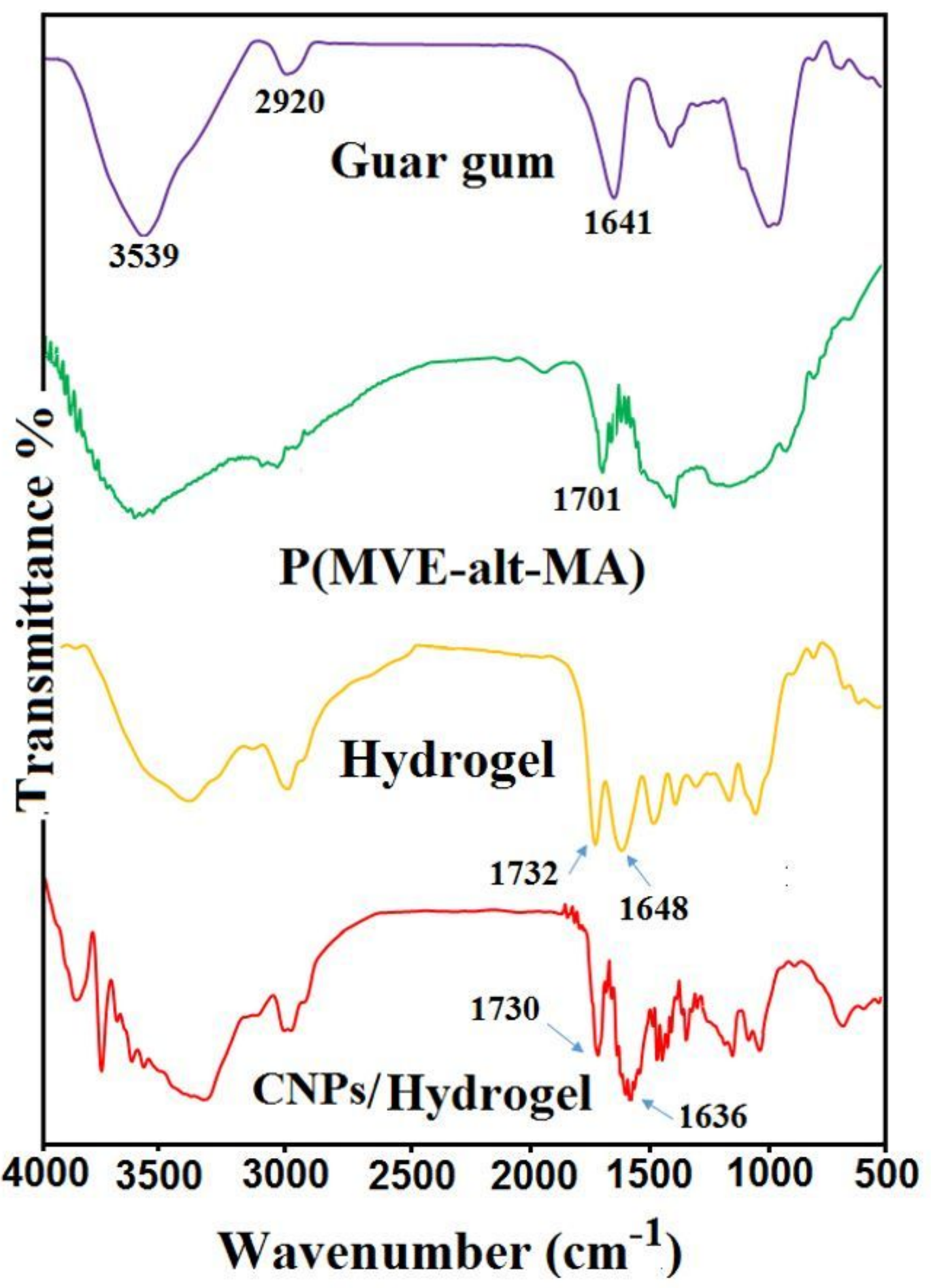

Figure 2

Fourier transform infrared (FTIR) spectra of Guar Gum (GG), PMVE-MAn, and GG/ PMVE-MA hydrogels (Hydrogel). 
GG/P20

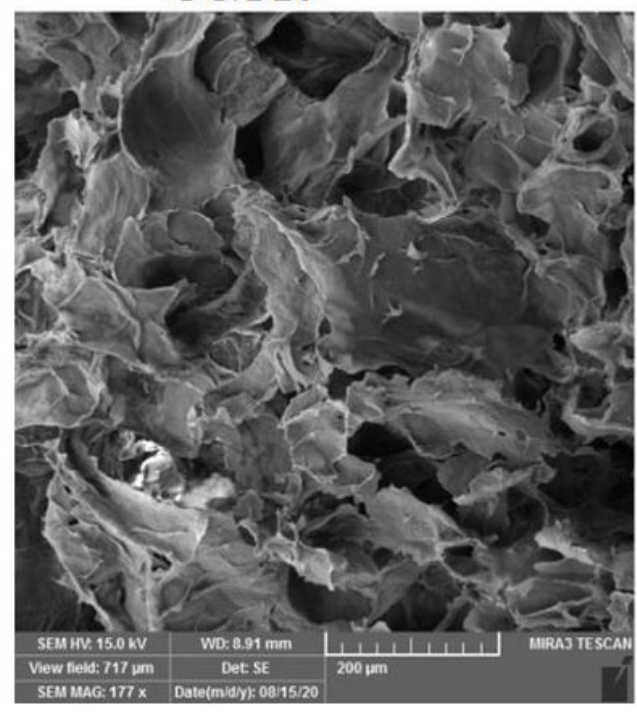

GG/P40

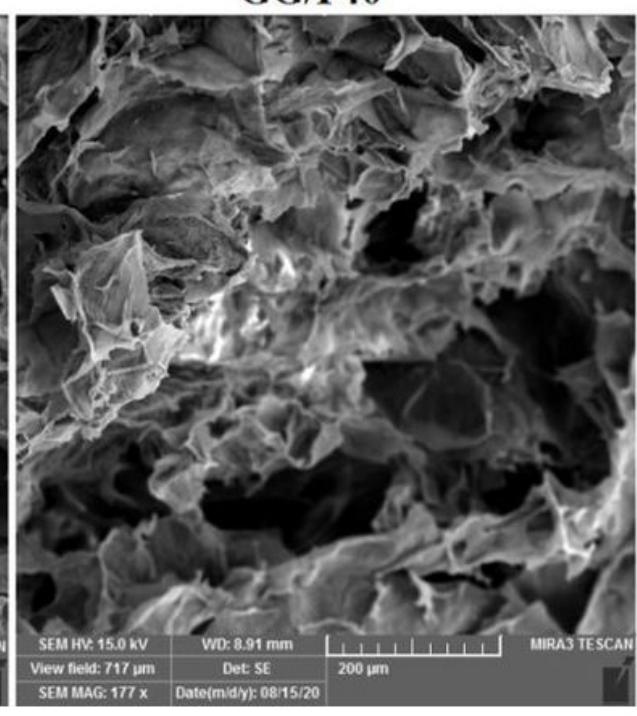

GG/P70

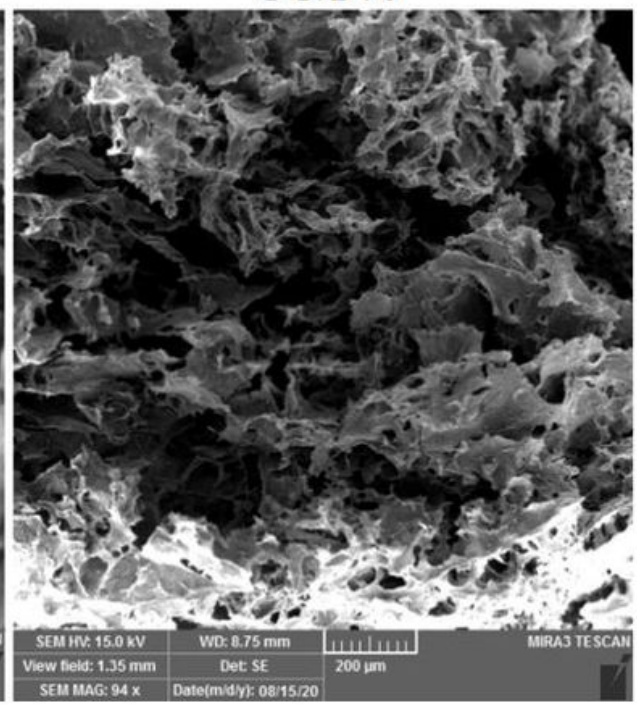

Figure 3

Scanning electron microscopy (SEM) images of Guar Gum/PMVE-MA (GG/P) hydrogels with various combination ratios of GG, and P(MVE-alt-MA) (GG/P20, GG/P40, and GG/P70).

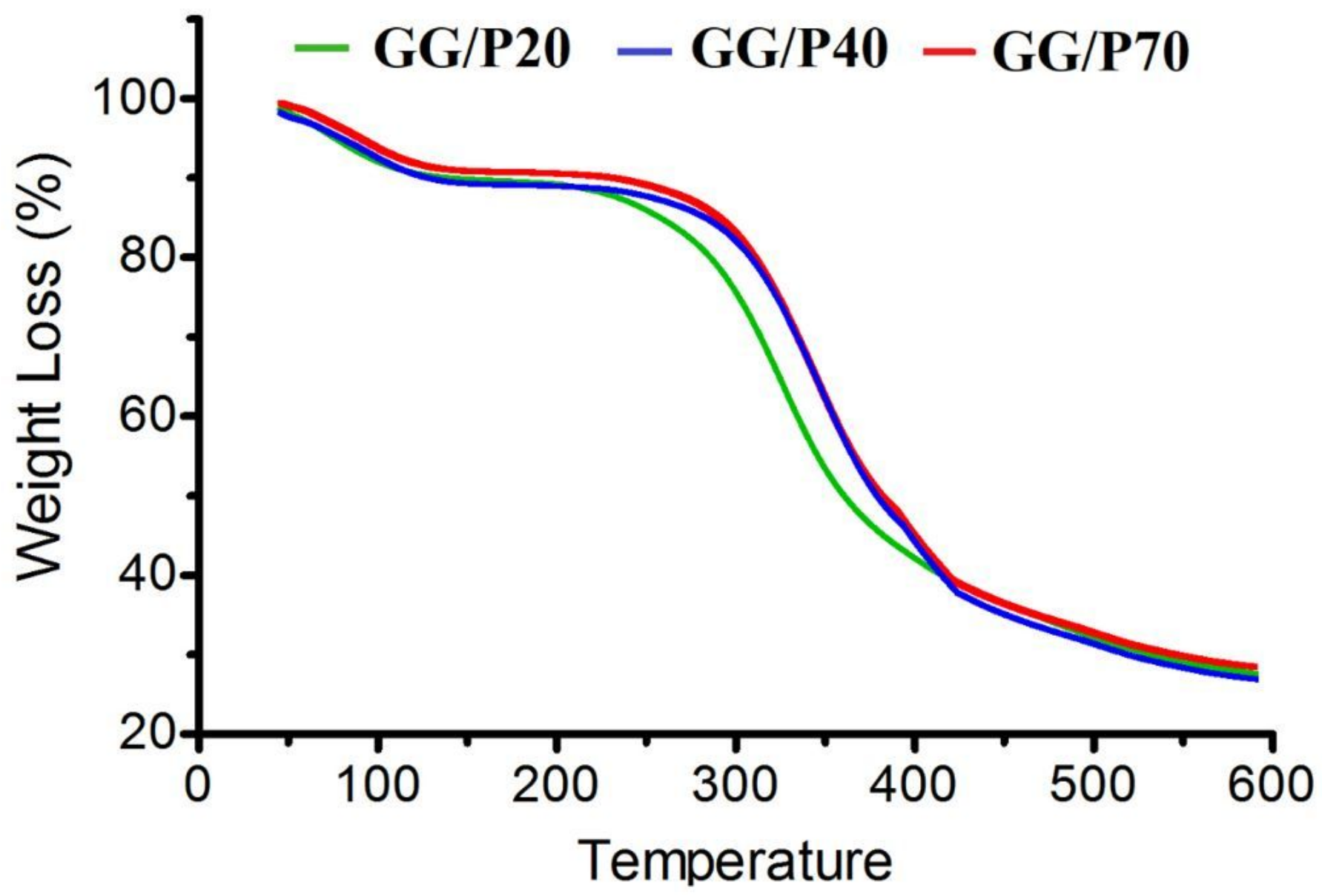


Figure 4

TGA thermograms of Guar Gum (GG)/PMVE-MA (P) hydrogels with various blending weight ratio of GG, and PMVE-MAn (GG/P20, GG/P40, and GG/P70).

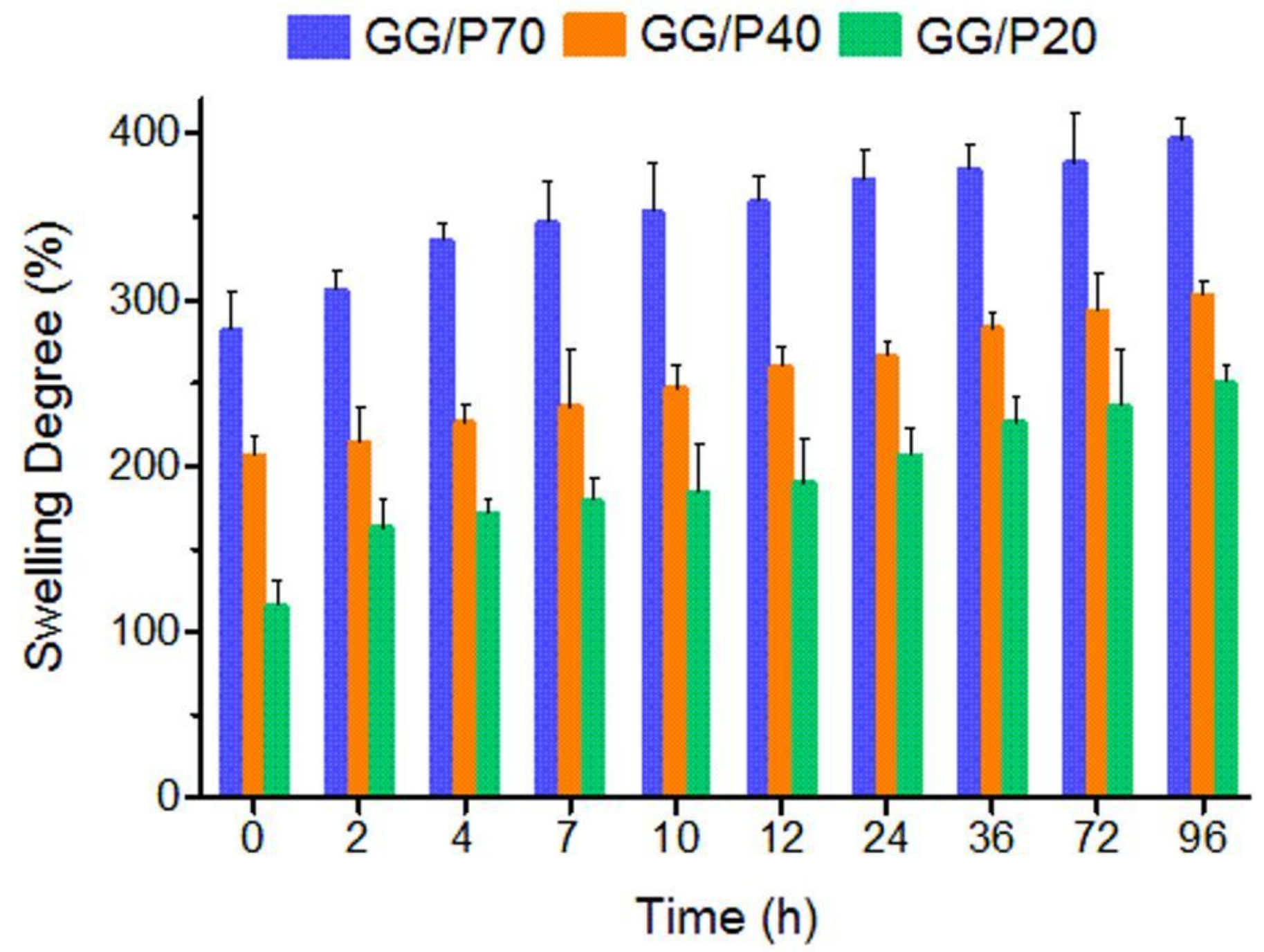

Figure 5

Swelling degree of Guar Gum (GG)/PMVE-MA (P) hydrogels with different blending weight ratios of GG and PMVE-MAn (GG/P20, GG/P40, and GG/P70). 

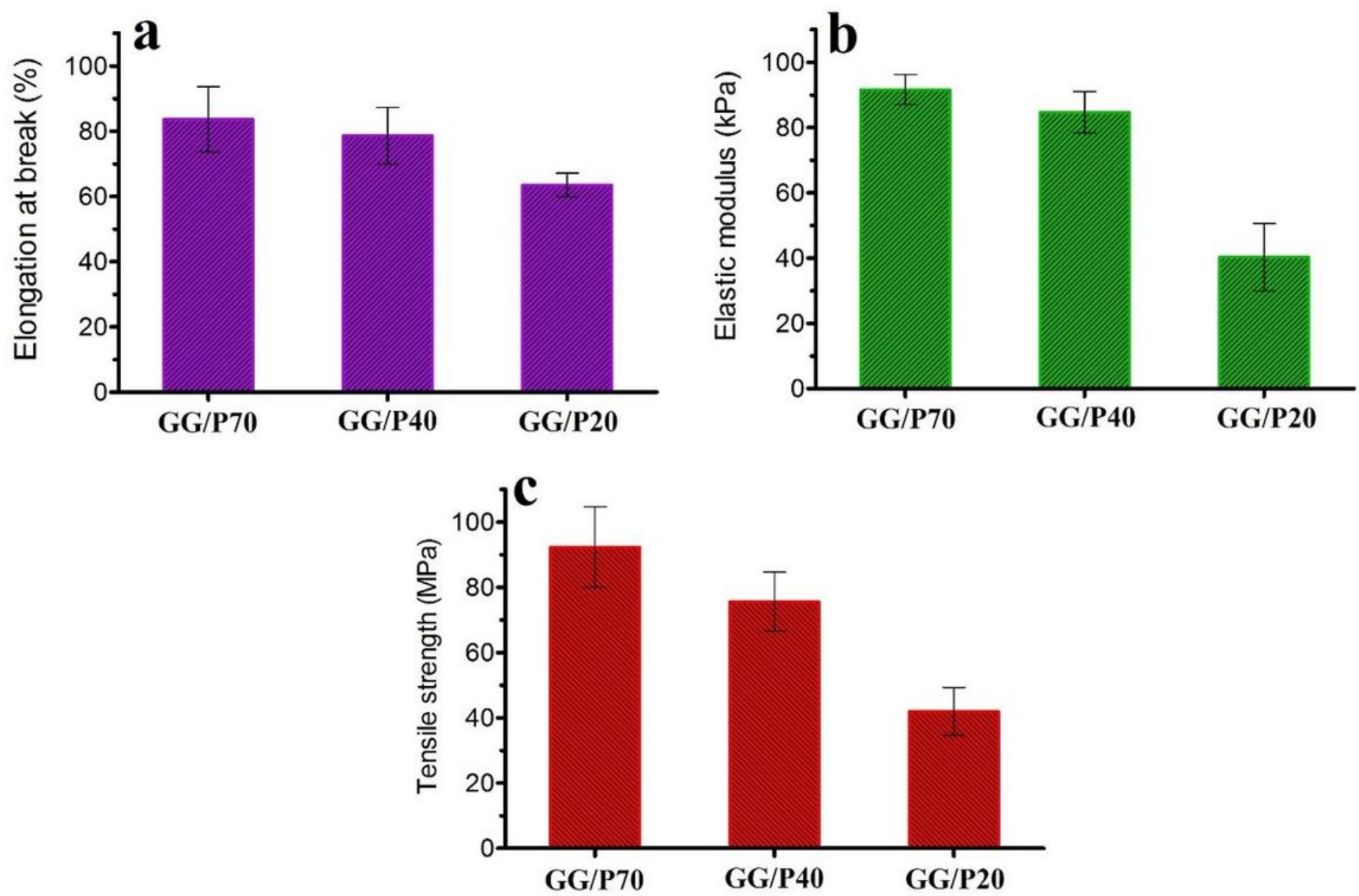

Figure 6

Mechanical characteristics of Guar Gum (GG)/PMVE-MA (P) hydrogels with different blending weight ratios of GG, and PMVE-MAn (GG/P20, GG/P40, and GG/P70) 


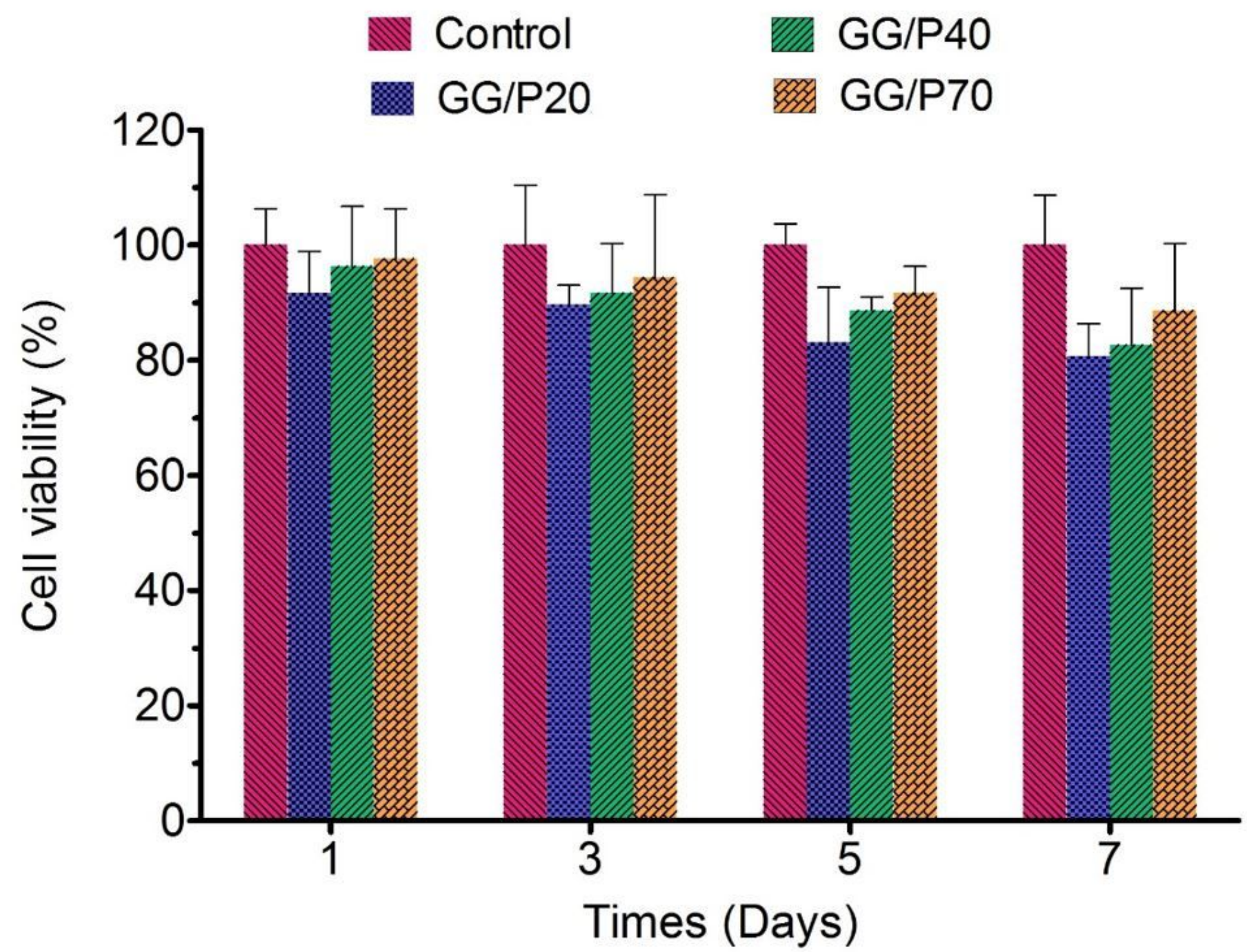

Figure 7

Cell viability of Guar Gum (GG)/PMVE-MA (P) with different blending weight ratio of GG, and PMVE-MAn (GG/P20, GG/P40, and GG/P70). 


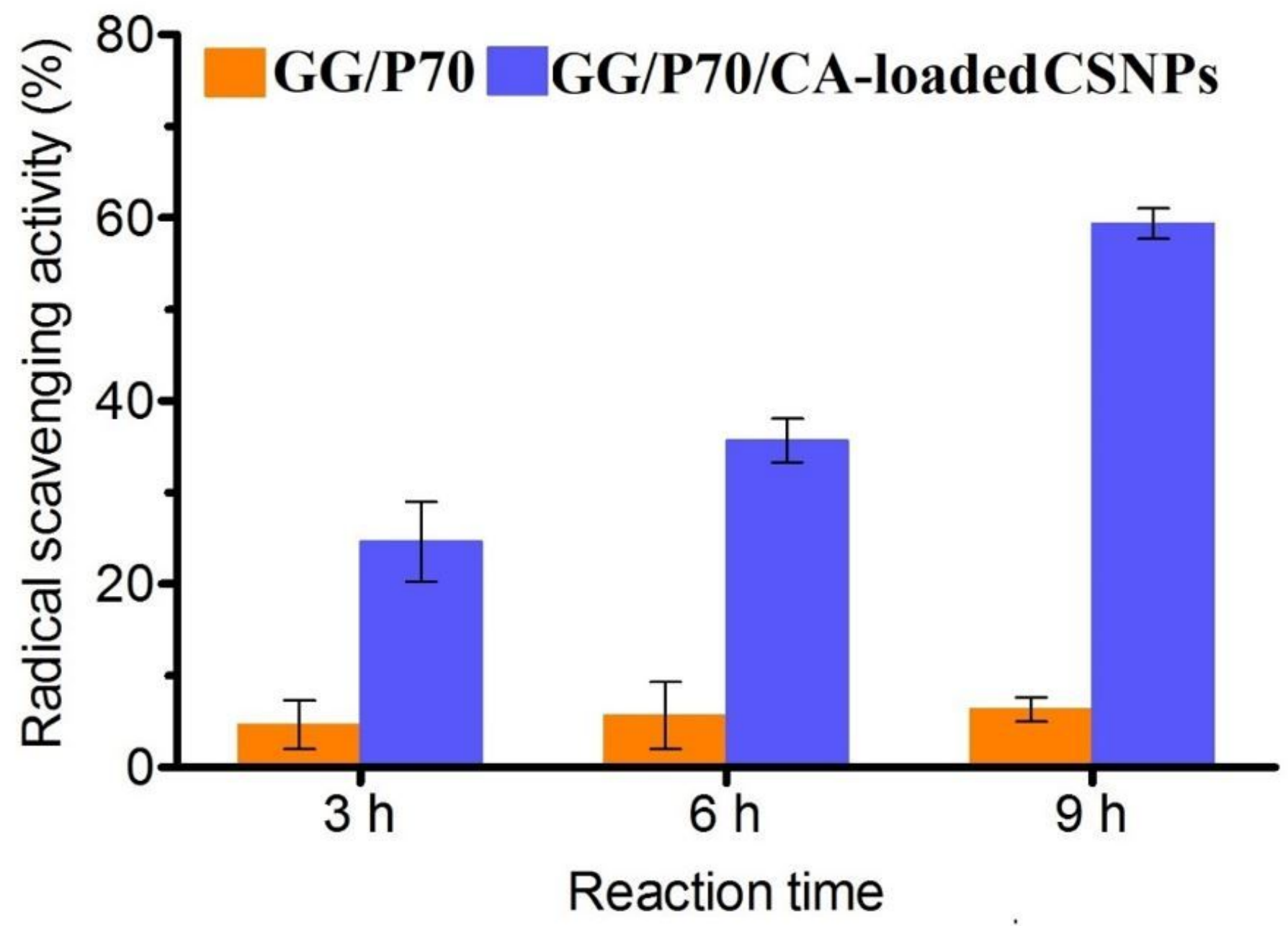

Figure 8

The DPPH radical scavenging activity of Guar Gum (GG)/PMVE-MA (P) with optimal formulation (GG/P70), and GG/P70 containing cinnamaldehyde-loaded chitosan nanoparticles (GG/P70/CA-loaded CSNPs) after different reaction times $(3,6$, and $9 \mathrm{~h})$. 


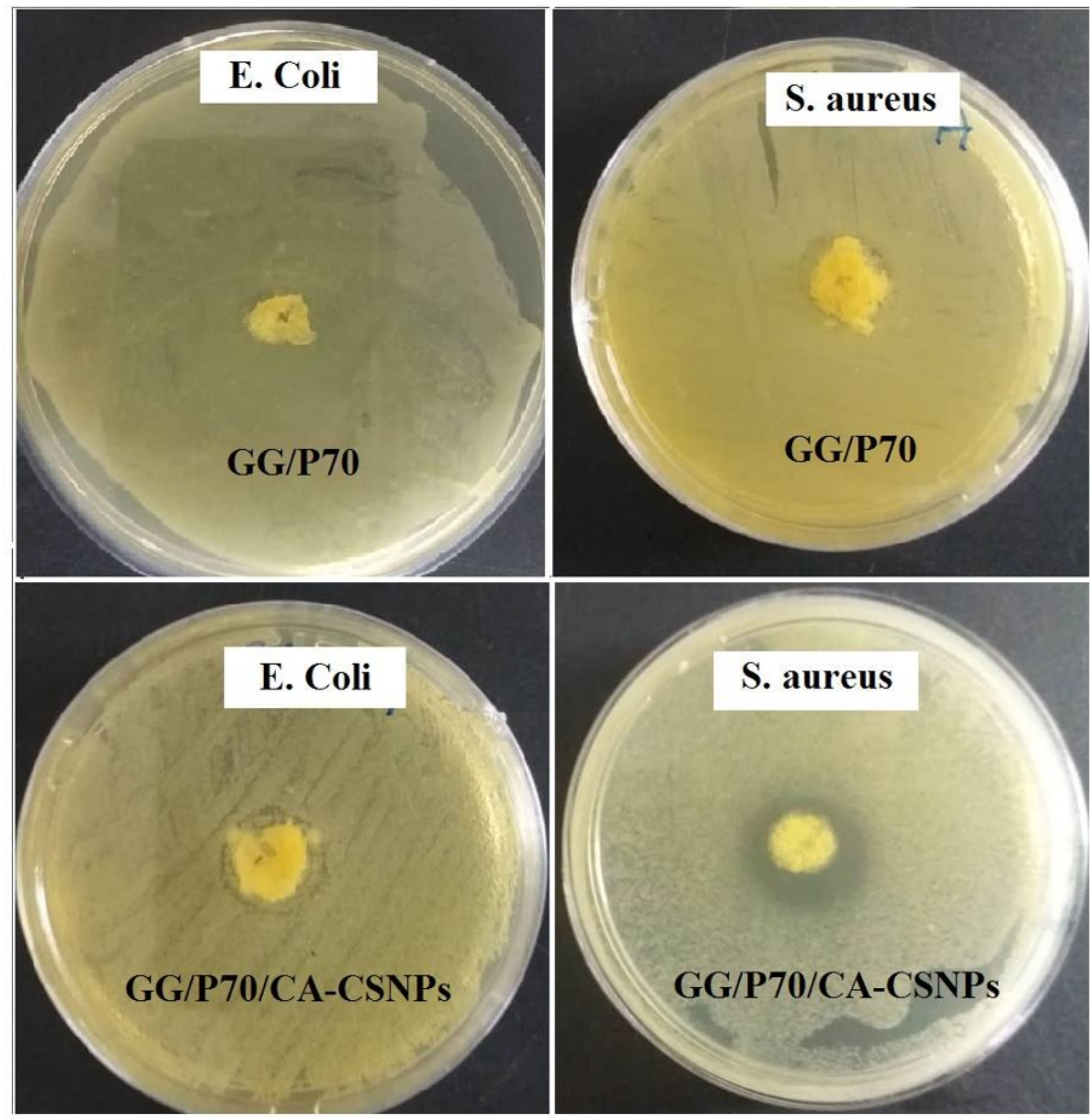

Figure 9

The Antibacterial activity of Guar Gum (GG)/PMVE-MA (P) with optimal formulation (GG/P70), and GG/P70 containing cinnamaldehyde-loaded chitosan nanoparticles (GG/P70/CA-loaded CSNPs) against E. coil, and S. areus bacteria. 